\title{
A time-dependent Parametrized Background Data-Weak approach
}

\author{
Amina Benaceur*
}

\begin{abstract}
This paper addresses model reduction with data assimilation by elaborating on the Parametrized Background Data-Weak (PBDW) approach [6] recently introduced to combine numerical models with experimental measurements. This approach is here extended to a timedependent framework by means of a POD-greedy reduced basis construction.
\end{abstract}

\section{Introduction}

The Parameterized-Background Data-Weak (PBDW) formulation for variational data assimilation is a data-driven reduced order modeling approach that was initially devised in [6] so as to merge prediction by model with prediction by data. The PBDW approach has been developed in order to estimate the true state $u^{\text {true }}$ of a physical system for several configurations. Supposing that the true state $u^{\text {true }}$ depends on some unknown parameter $\omega$ in an unknown parameter set $\Theta$ that represents the unanticipated uncertainty, the goal is to account for the dependency of the true state $u^{\text {true }}(\omega)$ on uncertain parameters by means of the sole knowledge of data. In this paper, whenever the context is unambiguous, the parameter $\omega$ is dropped.

The formulation combines a so-called 'best-knowledge' (bk) model represented by a parametrized partial differential equation (PDE) and experimentally observable measurements. The use of data in the PBDW approach is

*Amina Benaceur.

Massachusetts Institute of Technology, USA. University Paris-Est, CERMICS (ENPC) and INRIA Paris, France. EDF Lab Les Renardières, France. email: benaceur@mit.edu This work is supported by EDF and ANRT.

The author is thankful to A.T. Patera and A. Ern. 
fundamental not only to reconstruct the quantities of interest, but also to correct the possible bias in the mathematical bk model.

The PBDW approach was devised in [6] for steady problems. It has been subject to active research in recent years and it has been used for several applications. Among others, we mention [2], 3], [5], [7, 8], and [9]. To the author's knowledge, the related research in the literature remains in the steady framework. In this paper, we propose, as initiated in [1, an extension of the PBDW approach to time-dependent state estimation. We build appropriate background spaces for the time-dependent setting using the POD-greedy algorithm [4].

This paper is organized as follows. Section 2 introduces the notation. Section 3 extends the PBDW approach to the time-dependent framework and discusses the offline stage. Section 4 assesses the method via numerical experiments.

\section{Basic notation and best-knowledge (bk) mod- els}

We consider a spatial domain (open, bounded, connected subset) $\Omega \subset \mathbb{R}^{d}$, $d \geqslant 1$, with a Lipschitz boundary. We introduce a Hilbert space $\mathcal{U}$ composed of functions defined over $\Omega$. The space $\mathcal{U}$ is endowed with an inner product $(\cdot, \cdot)$ and we denote by $\|\cdot\|$ the induced norm; $\mathcal{U}$ consists of functions $\{w: \Omega \rightarrow$ $\mathbb{R} \mid\|w\|<\infty\}$. To fix the ideas, we assume that $H_{0}^{1}(\Omega) \subset \mathcal{U} \subset H^{1}(\Omega)$, and we denote the dual space of $\mathcal{U}$ by $\mathcal{U}^{\prime}$. The Riesz operator $R_{\mathcal{U}}: \mathcal{U}^{\prime} \rightarrow \mathcal{U}$ satisfies, for each $\ell \in \mathcal{U}^{\prime}$, and for all $v \in \mathcal{U}$, the equality $\left(R_{\mathcal{U}}(\ell), v\right)=\ell(v)$. Finally, we introduce a parameter set $\mathcal{P} \subset \mathbb{R}^{p}, p \geqslant 1$, whose elements are generically denoted by $\mu \in \mathcal{P}$.

The first source of information we shall afford ourselves in the PBDW approach is a so-called 'best-knowledge' (bk) mathematical model in the form of a parameterized PDE posed over the domain $\Omega$. Given a parameter value $\mu$ in the parameter set $\mathcal{P}$, we denote the solution to the bk parameterized PDE as $u^{\mathrm{bk}}(\mu) \in \mathcal{U}$. Then, the manifold associated with the solutions of the bk model is $\mathcal{M}^{\mathrm{bk}}:=\left\{u^{\mathrm{bk}}(\mu) \mid \mu \in \mathcal{P}\right\} \subset \mathcal{U}$. In ideal situations, the true solution $u^{\text {true }}$ is well approximated by the bk manifold, i.e., the model error $\epsilon_{\bmod }^{\mathrm{bk}}\left(u^{\text {true }}\right):=\inf _{z \in \mathcal{M}^{\mathrm{bk}}}\left\|u^{\text {true }}-z\right\|$ is very small.

We introduce nested background subspaces $\mathcal{Z}_{1} \subset \ldots \subset \mathcal{Z}_{N} \subset \ldots \subset \mathcal{U}$ that are generated to approximate the bk manifold $\mathcal{M}^{\text {bk }}$ to a certain accuracy. These subspaces can be built using various model-order reduction techniques, for instance, the Reduced Basis (RB) method. The indices of the subspaces 
conventionally indicate their dimensions. To measure how well the true solution is approximated by the background space $\mathcal{Z}_{N}$, we define the quantity $\epsilon_{N}^{\mathrm{bk}}\left(u^{\text {true }}\right):=\inf _{z \in \mathcal{Z}_{N}}\left\|u^{\text {true }}-z\right\|$. Although $N$ is large enough, $\epsilon_{N}^{\text {bk }}\left(u^{\text {true }}\right)$ does not tend to zero since $u^{\text {true }}$ rarely lies in $\mathcal{M}^{\text {bk }}$ in realistic engineering study cases.

\section{Time-dependent PBDW}

Consider a finite time interval $I=[0, T]$, with $T>0$. To discretize in time, we consider an integer $K \geqslant 1$, we define $0=t^{0}<\cdots<t^{K}=T$ as $(K+1)$ distinct time nodes over $I$, and we set $\mathbb{K}^{\text {tr }}=\{1, \ldots, K\}, \overline{\mathbb{K}}^{\text {tr }}=\{0\} \cup \mathbb{K}^{\text {tr }}$ and $I^{\text {tr }}=\left\{t^{k}\right\}_{k \in \overline{\mathbb{K}}^{\mathrm{tr}}}$. We aim at deriving a state estimate for a time-dependent solution in the framework illustrated in Figure 1 .

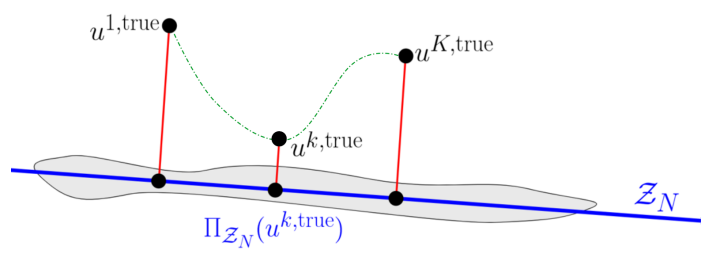

Figure 1: Characterization of the bk model in a time-dependent context.

\subsection{Limited-observations statement}

Assuming that $u^{\text {true }} \in L^{1}(I ; \mathcal{U})$, we introduce the time-integration intervals $\mathcal{I}^{k}=\left[t^{k}-\delta t_{k}, t^{k}+\delta t_{k}\right]$, for all $k \in \mathbb{K}^{\text {tr }}$, where $\delta t^{k}>0$ is a parameter related to the precision of the sensor (ideally, $\delta t^{k}<\min \left(t^{k+1}-t^{k}, t^{k}-t^{k-1}\right)$ with obvious adaptation if $\mathrm{k}=\mathrm{K})$. Then, for any function $v \in L^{1}(I ; \mathcal{U})$, we define the time-averaged snapshots

$$
v^{k}(x):=\frac{1}{\left|\mathcal{I}^{k}\right|} \int_{\mathcal{I}^{k}} v(t, x) d t \in \mathcal{U}, \quad \forall k \in \mathbb{K}^{\mathrm{tr}} .
$$

We consider observation functionals that render the behavior of given sensors. These functionals act on time-averaged snapshots of the true solution, i.e., we consider

$$
\ell_{m}^{k, \text { obs }}\left(u^{\text {true }}\right):=\ell_{m}^{\text {obs }}\left(u^{k, \text { true }}\right), \quad \forall m \in\{1, \ldots, M\}, \forall k \in \mathbb{K}^{\text {tr }} .
$$


We then introduce the time-independent observable space $\mathcal{U}_{M}=\operatorname{Span}\left\{q_{1}, \ldots, q_{M}\right\} \subset$ $\mathcal{U}$. The observation functionals in $\mathcal{U}^{\prime}$ are then defined as

$$
\ell_{m}^{k, \text { obs }}\left(u^{\text {true }}\right)=\left(u^{k, \text { true }}, q_{m}\right), \quad \forall m \in\{1, \ldots, M\}, \quad \forall k \in \mathbb{K}^{\text {tr }} .
$$

For fixed sensor locations, the computational effort to compute the Riesz representations of the observation functionals is time-independent and is incurred only once, so that the experimental observations satisfy $\ell_{m}^{k, \text { obs }}\left(u^{\text {true }}\right)=$ $\frac{1}{\left|\mathcal{I}_{k}\right|} \int_{\mathcal{I}^{k}} \ell_{m}^{\text {obs }}\left(u^{\text {true }}(t, \cdot)\right) d t$, for all $m \in\{1, \ldots, M\}$ and $k \in \mathbb{K}^{\text {tr }}$.

We are now ready to write the limited-observations PBDW statement: for each $k \in \mathbb{K}^{\operatorname{tr}}$, find $\left(u_{N, M}^{k, *}, z_{N, M}^{k, *}, \eta_{N, M}^{k, *}\right) \in \mathcal{U} \times \mathcal{Z}_{N} \times \mathcal{U}$ such that

$$
\left(u_{N, M}^{k, *}, z_{N, M}^{k, *}, \eta_{N, M}^{k, *}\right)=\underset{\substack{u_{N, M} \in \mathcal{U} \\ z_{N, M} \in \mathcal{Z}_{N} \\ \eta_{N, M} \in \mathcal{U}}}{\operatorname{arginf}}\left\|\eta_{N, M}\right\|,
$$

subject to

$$
\begin{array}{llrl}
\left(u_{N, M}, v\right) & =\left(\eta_{N, M}, v\right)+\left(z_{N, M}, v\right), & & \forall v \in \mathcal{U}, \\
\left(u_{N, M}, \phi\right) & =\left(u^{k, \text { true }}, \phi\right), & & \forall \phi \in \mathcal{U}_{M} .
\end{array}
$$

The limited-observations saddle-point problem associated with (4) reads: for each $k \in \mathbb{K}^{\mathrm{tr}}$, find $\left(z_{N, M}^{k, *}, \eta_{N, M}^{k, *}\right) \in \mathcal{Z}_{N} \times \mathcal{U}_{M}$ such that

$$
\begin{aligned}
\left(\eta_{N, M}^{k, *}, q\right)+\left(z_{N, M}^{k, *}, q\right) & =\left(u^{k, \text { true }}, q\right), & & \forall q \in \mathcal{U}_{M}, \\
\left(\eta_{N, M}^{k, *}, p\right) & =0, & & \forall p \in \mathcal{Z}_{N},
\end{aligned}
$$

and the limited-observations state estimate is

$$
u_{N, M}^{k, *}=z_{N, M}^{k, *}+\eta_{N, M}^{k, *}, \quad \forall k \in \mathbb{K}^{\operatorname{tr}} .
$$

We use the following terminology. The PBDW statement (4)-(5) estimates the true state $u^{k, \text { true }}$. Thus, the solution $u_{N, M}^{k, *}$ is called the 'state estimate'. The first contribution $z_{N, M}^{k, *}$ in (7) lies in the background space $\mathcal{Z}_{N}$. Hence, $z_{N, M}^{k, *}$ is called the 'deduced background estimate'. The second contribution $\eta_{N, M}^{k, *}$ in (7) is brought by the inclusion of the observations in the PBDW statement. The observations supplement the bk model. Thus, $\eta_{N}^{k, *}$ is called the 'update estimate'. We highlight that the saddle-point problem 6 is well posed if and only if the stability constant $\beta_{N, M}$ satisfies

$$
\beta_{N, M}:=\inf _{w \in \mathcal{Z}_{N}} \sup _{v \in \mathcal{U}_{M}} \frac{(w, v)}{\|w\|\|v\|} \in(0,1]
$$


The deduced background estimate $z_{N}^{k, *}$ can only represent anticipated uncertainty. Since the bk model is often deficient, one cannot realistically assume that the state estimate $u_{N}^{k, *}$ of $u^{k \text {,true }}$ lies completely in the bk manifold. Therefore, the update estimate $\eta_{N}^{k, *}$ is meant to cure the deficiency of the bk model by capturing unanticipated uncertainty. The key idea of the PBDW statement (4)-(5) is to search for the smallest correction to the bk manifold.

The saddle-point problem (6) is purely geometric and does not include any explicit reference to the bk model since the unique link to the bk model is through the background space $\mathcal{Z}_{N}$. This non-intrusiveness of (6) simplifies its implementation and makes the PBDW approach applicable to a wide class of engineering problems.

Remark 1 (Pointwise measurements). For simplicity of implementation, assuming that $u^{\text {true }} \in \mathcal{C}^{0}(I ; \mathcal{U})$, one may consider pointwise measurements in time, i.e., $\left(u^{k \text {,true }}, q_{m}\right)=\ell_{m}^{\text {obs }}\left(u^{\text {true }}\left(t^{k}, \cdot\right)\right)$, for all $m \in\{1, \ldots, M\}$ and $k \in \mathbb{K}^{\text {tr }}$. This assumption is typically reasonable for a sensor of small precision $\delta t^{k}$.

In algebraic form, the limited-observations PBDW statement reads: for each $k \in \mathbb{K}^{\text {tr }}$, find $\left(\mathbf{z}^{k, *}, \boldsymbol{\eta}^{k, *}\right) \in \mathbb{R}^{N} \times \mathbb{R}^{M}$ such that

$$
\left(\begin{array}{cc}
\mathbf{A} & \mathbf{B} \\
\mathbf{B}^{T} & \mathbf{0}
\end{array}\right)\left(\begin{array}{l}
\boldsymbol{\eta}^{k, *} \\
\mathbf{z}^{k, *}
\end{array}\right)=\left(\begin{array}{c}
\boldsymbol{\ell}^{k, \mathrm{obs}} \\
\mathbf{0}
\end{array}\right)
$$

with the matrices $\mathbf{A}=\left(\left(q_{m^{\prime}}, q_{m}\right)\right)_{m, m^{\prime}} \in \mathbb{R}^{M \times M}$ and $\mathbf{B}=\left(\left(\zeta_{n}, q_{m}\right)\right)_{m, n} \in \mathbb{R}^{M \times N}$, and the vector of observations $\ell^{k, \text { obs }}=\left(\ell_{m}^{\text {obs }}\left(u^{k, \text { true }}\right)\right)_{m} \in \mathbb{R}^{M}$. We solve (9) through an offline/online decomposed computational procedure whenever several realizations $u^{\text {true }}(\omega)$ of the true state are to be considered.

Remark 2 (PBDW matrices). Notice that the PBDW matrices $\mathbf{A}$ and $\mathbf{B}$ are time-independent; only the right-hand side in (9) depends on $k$.

\subsection{Offline stage}

The main goal is to address the construction of the background space $\mathcal{Z}_{N}$. Suppose that we have computed a set of High Fidelity (HF) trajectories $\mathcal{S}=$ $\left(\mathcal{S}_{k}\right)_{k \in \mathbb{K}^{\operatorname{tr}}}=\left(\left(u^{k}(\mu)\right)_{\mu \in \mathcal{P}^{\text {tr }}}\right)_{k \in \mathbb{K}^{\text {tr }}}$, where $u^{k}(\mu):=u(\mu)\left(t^{k}, \cdot\right)$, for all $k \in \mathbb{K}^{\text {tr }}$. If we were to consider the PBDW statement (4)-(5) for each $k \in \mathbb{K}^{\text {tr }}$ as an independent steady PBDW statement, we would be using the time-dependent background spaces $\mathcal{Z}_{N^{k}}^{k}=\operatorname{POD}\left(\mathcal{S}_{k}, \epsilon_{\mathrm{POD}}\right)$, for all $k \in \mathbb{K}^{\mathrm{tr}}$, where the procedure POD refers to the Proper Orthogonal Decomposition of the set $\mathcal{S}_{k}$ with a truncation threshold $\epsilon_{\mathrm{POD}}$. Yet, this strategy is not convenient since the sizes $N^{k}$ of the background spaces $\mathcal{Z}_{N^{k}}^{k}$ would depend on $k$. Since the observable space 
$\mathcal{U}_{M}$ is fixed, the same non-homogeneity between time nodes would also arise in the stability constant $\beta_{N^{k}, M}$. Thus, we propose to apply a POD-greedy algorithm [4] to build a time-independent background space $\mathcal{Z}_{N}$ that will be used for all $k \in \mathbb{K}^{\text {tr }}$. The advantage is that the PBDW matrices $\mathbf{A}$ and $\mathbf{B}$ and the stability constant $\beta_{N, M}$ remain unchanged regardless of the discrete time node. The offline stage using the POD-greedy algorithm is summarized in Algorithm 1 .

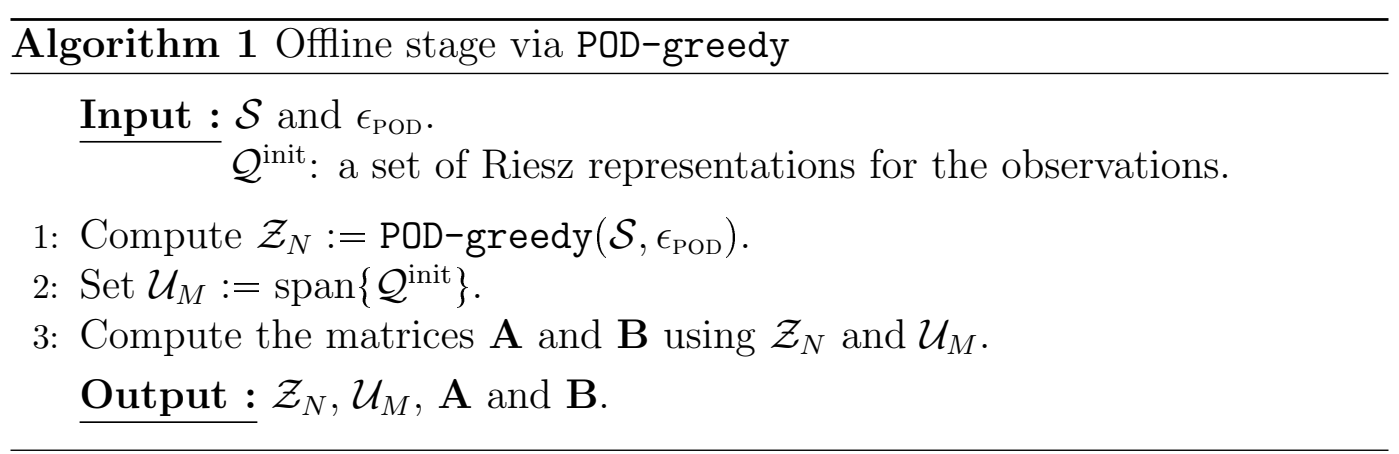

\section{Numerical results}

In this section, we illustrate the above developments on a test case related to the heat equation. We consider a two-dimensional setting based on the plate
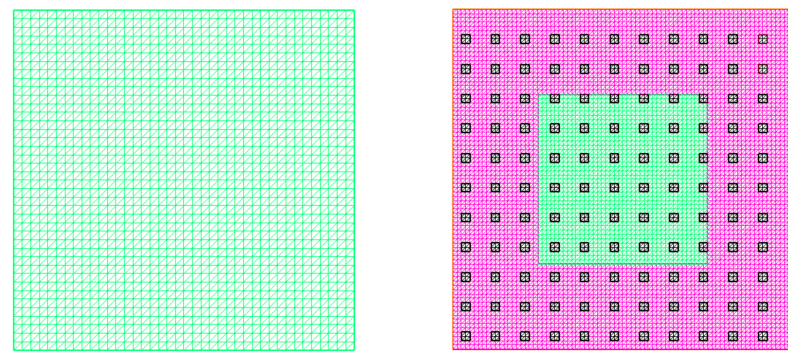

Figure 2: Computational domain and mesh with $\mathcal{N}=6561$. The little black squares are observation subsets $\left\{\mathcal{R}_{m}\right\}_{m=1}^{121}$. Left: Mono-material plate corresponding to the mathematical model. Right: Bi-material plate corresponding to the physical reality.

illustrated in the left panel of Figure 2 with $\Omega=(-2,2)^{2} \subset \mathbb{R}^{2}$. We use a finite element subspace $\mathcal{U}^{\mathcal{N}} \subset \mathcal{U}=H^{1}(\Omega)$ consisting of continuous, piecewise affine functions in order to generate HF trajectories. The FEM subspace $\mathcal{U}^{\mathcal{N}}$ 
is based on a mesh that contains $\mathcal{N}=6561$ nodes. The experimental data is generated synthetically and the observation subsets $\left\{\mathcal{R}_{m}\right\}_{1 \leqslant m \leqslant M}$ are uniformly selected over the plate as illustrated in the right panel of Figure 2. Regarding implementation, the HF computations use the software FreeFem++, whereas the reduced-order modeling and the PBDW-related algorithms have been developed in Python. We address the following parabolic PDE with nonlinear Stefan-Boltzmann boundary conditions: For many values of the parameter $\mu \in \mathcal{P}$, find $u(\mu): I \times \Omega \rightarrow \mathbb{R}$ such that

$$
\left\{\begin{aligned}
\frac{\partial u(\mu)}{\partial t}-\nabla \cdot(D(\mu) \nabla u(\mu)) & =0, & & \text { in } I \times \Omega, \\
u(\mu)(t=0, \cdot) & =u_{0}, & & \text { in } \Omega, \\
-D(\mu) \frac{\partial u}{\partial n} & =\sigma \varepsilon\left(u^{4}-u_{r}^{4}\right), & & \text { on } I \times \partial \Omega,
\end{aligned}\right.
$$

where $u_{0}=293.15 \mathrm{~K}\left(20^{\circ} \mathrm{C}\right)$. The Stefan-Boltzmann boundary condition on $\partial \Omega$ is defined using an enclosure temperature $u_{r}=303.15 \mathrm{~K}\left(30^{\circ} \mathrm{C}\right)$, the StefanBoltzmann constant $\sigma=5.67 \times 10^{-8} \mathrm{~W} \cdot \mathrm{m}^{-2} \cdot \mathrm{K}^{-4}$, and an emissivity $\varepsilon=3.10^{-3}$. Regarding time discretization, we consider the time interval $I=[0,10] \mathrm{s}$, the set of discrete times nodes $\mathbb{K}^{\text {tr }}=\{1, \ldots, 200\}$, and a constant time step $\Delta t^{k}=0.1 \mathrm{~s}$ for all $k \in \mathbb{K}^{\text {tr }}$. We also define the parameter interval $\mathcal{P}=[0.1,2]$ and the set $\mathcal{P}^{\operatorname{tr}}=\{0.1 i, 1 \leqslant i \leqslant 20\}$.

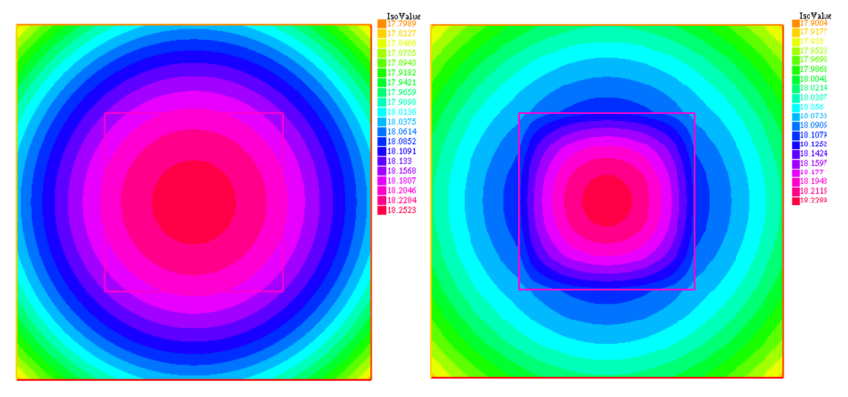

Figure 3: Left: HF solution for the bk model (values from $17.80^{\circ} \mathrm{C}$ to $18.25^{\circ} \mathrm{C}$ ). Right: Synthetic true solution using a bi-material plate (values from $17.90^{\circ} \mathrm{C}$ to $18.23^{\circ} \mathrm{C}$ ).

The background spaces $\mathcal{Z}_{N}$ will be generated by solving the nonlinear PDE (10) with a uniform diffusivity function $D(\mu)$ such that for all $x \in \Omega$, $D(\mu)(x)=D_{\text {uni }}(\mu)(x):=\mu \mathbf{1}_{\Omega}(x)$. The HF bk solutions and the true solutions are respectively displayed in the left and right panels of Figure 3 . The temperature profile for the true solution over the bi-material plate at the end of the simulation, i.e., at $t^{K}=10 \mathrm{~s}$, clearly shows a different behavior at the boundaries of the inner material. 
Using the weighted $H^{1}$-norm, we define the state estimation relative $H^{1}$ error $e^{k}(\mu)$ as

$$
e^{k}(\mu):=\frac{\left\|u^{k, \text { true }}(\mu)-u_{N, M}^{k, *}(\mu)\right\|_{H^{1}(\Omega)}}{\left\|u^{k, \text { true }}(\mu)\right\|_{H^{1}(\Omega)}}, \quad \forall \mu \in \mathcal{P} .
$$

Figure 4 shows the relative $H^{1}$-error $e^{k}(\mu)$ defined in (11) using $M=121$ observations to build the observable space $\mathcal{U}_{M}$. For $\epsilon_{\mathrm{POD}}=10^{-4}, \mathcal{Z}_{N}$ is spanned by $N=7$ vectors. Notice that the error vanishes for $\mu=0.25$ since this configuration is equivalent to a perfect bk model, meaning that the mathematical model coincides with the physical reality. We notice that the relative $H^{1}$-error $e^{k}(\mu)$ increases on the right panel of Figure 4 because the stability constant decreases. Figure 5 visualizes the relative $H^{1}$-error $e^{k}(\mu)$ for a higher number
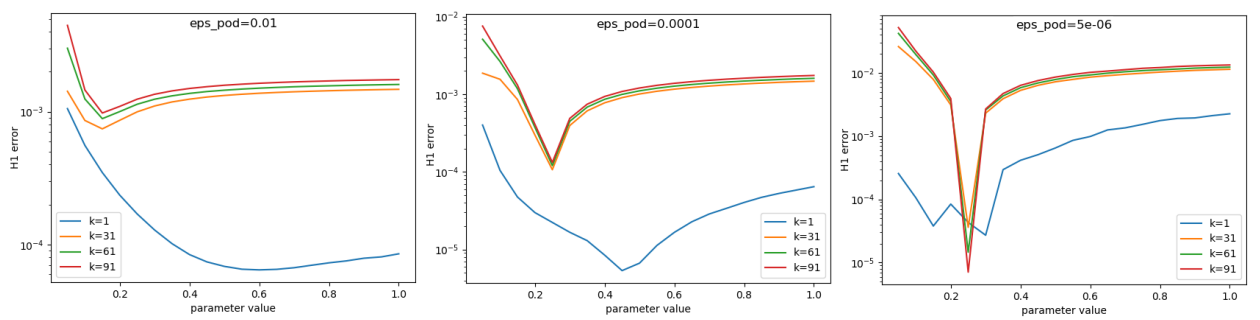

Figure 4: Relative $H^{1}$-error $e^{k}(\mu)$ for some time nodes $k \in \mathbb{K}^{\text {tr }}$ and $M=121$. Left: $\epsilon_{\mathrm{POD}}=10^{-2}(N=3)$. Middle: $\epsilon_{\mathrm{POD}}=10^{-4}$ $(N=7)$. Right: $\epsilon_{\mathrm{POD}}=10^{-6}(N=11)$.

of observations $M=676$. We observe that augmenting the dimension of the observable space $\mathcal{U}_{M}$ cures the stability issue. Also, the errors are lower owing to the higher number of observations. Finally, Figure 6 shows the stability
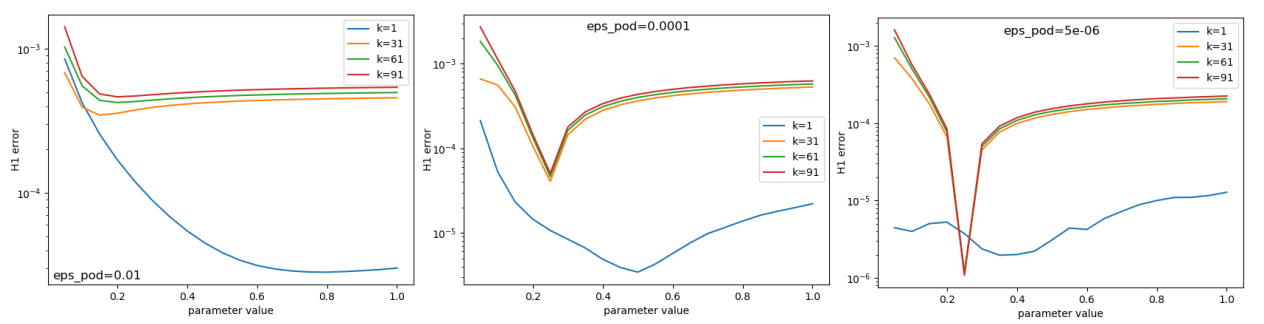

Figure 5: Relative $H^{1}$-error $e^{k}(\mu)$ for some time nodes $k \in \mathbb{K}^{\text {tr }}$ and $M=676$. Left: $\epsilon_{\mathrm{POD}}=10^{-2}(N=3)$. Middle: $\epsilon_{\mathrm{POD}}=10^{-4}$ $(N=7)$. Right: $\epsilon_{\mathrm{POD}}=5 \cdot 10^{-6}(N=11)$.

constant $\beta_{N, M}$ as a function of the number of observations $M$. The nonlinear 
character of the problem does not influence the overall features of the PBDW statement since previous linear tests in the literature have shown a similar behavior. This observation corroborates the independence of the saddle-point problem (6) with regard to the bk model.
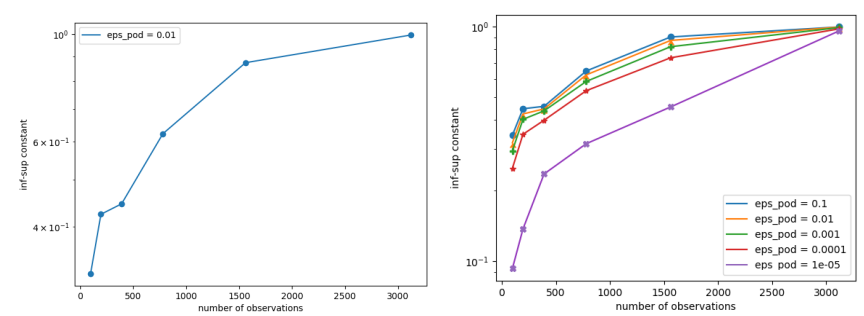

Figure 6: Stability constant $\beta_{N, M}$. On the right panel, the values of $N$ are respectively $2,3,5,7,11$ for the values of $\epsilon_{\mathrm{POD}}$ in decreasing order.

\section{References}

[1] A. Benaceur. Model reduction for nonlinear problems in thermics and mechanics. Thesis, Université Paris-Est Marne la Vallée, 2018.

[2] F. Galarce, J.-F. Gerbeau, D. Lombardi, and O. Mula. State estimation with nonlinear reduced models. Application to the reconstruction of blood flows with Doppler ultrasound images. arXiv e-prints, page arXiv:1904.13367, Apr 2019.

[3] H. Gong, Y. Maday, O. Mula, and T. Taddei. PBDW method for state estimation: error analysis for noisy data and nonlinear formulation. arXiv e-prints, page arXiv:1906.00810, Jun 2019.

[4] B. Haasdonk. Convergence rates of the POD-greedy method. ESAIM Math. Model. Numer. Anal., 47(3):859-873, 2013.

[5] J. K. Hammond, R. Chakir, F. Bourquin, and Y. Maday. PBDW: A nonintrusive Reduced Basis Data Assimilation method and its application to an urban dispersion modeling framework. Appl. Math. Model., 76:1-25, 2019 .

[6] Y. Maday, A. T. Patera, J. D. Penn, and M. Yano. A parameterizedbackground data-weak approach to variational data assimilation: formulation, analysis, and application to acoustics. Internat. J. Numer. Methods Engrg., 102(5):933-965, 2015. 
[7] Y. Maday and T. Taddei. Adaptive PBDW Approach to State Estimation: Noisy Observations; User-Defined Update Spaces. SIAM J. Sci. Comput., 41(4):B669-B693, 2019.

[8] T. Taddei and A. T. Patera. A localization strategy for data assimilation; application to state estimation and parameter estimation. SIAM J. Sci. Comput., 40(2):B611-B636, 2018.

[9] T. Taddei, J. D. Penn, and A. T. Patera. Validation by Monte Carlo sampling of experimental observation functionals. Internat. J. Numer. Methods Engrg., 112(13):2135-2150, 2017. 